\title{
ON THE GLOBAL BEHAVIOR OF DIFFERENTIAL EQUATIONS ON TWO-DIMENSIONAL MANIFOLDS ${ }^{1}$
}

\author{
FELIX HAAS
}

The following is concerned with the in-the-large behavior of characteristics of differential equations on closed orientable manifolds. $C^{+}$ will denote a positive semi-characteristic, $\bar{C}$ the set of $\omega$-limit ${ }^{2}$ points of $C^{+}, V$ the vector field defining the differential equation, $M$ the manifold under consideration, and $W$ the vector field orthogonal to $V$. In all that follows we shall assume that $M$ is two-dimensional, closed and orientable. The following theorem holds:

THEOREM. If the vector field $V$ satisfies a Lipschitz condition and has at most a denumerable number of singular points, and if, for some $\mathrm{C}^{+}$, $\vec{C}$ does not contain any singular points, then either $M$ is a torus and $V$ is free of singular points or $\bar{C}$ is nowhere dense on $M$.

REMARK. If $M$ is a torus and $V$ is free of singular points then the work of Denjoy [2] shows that $\bar{C}$ may be dense on $M$.

Notation. If $p$ is a point of $M, C_{p}^{+}, C_{\bar{p}}^{-}, D_{p}^{+}$, and $D_{\bar{p}}^{-}$will denote the positive semi-characteristic of $V$, the negative semi-characteristic of $V$, the positive semi-characteristic of $W$, and the negative semicharacteristic of $W$ respectively. If we are given a directed arc from $A$ to $B$ on $M$ carrying a parameter $\rho$ and if $q$ is a point with parametric value $\rho$, then we shall use $C_{\rho}^{+}, C_{\rho}^{-}, D_{\rho}^{+}$, and $D_{\rho}^{-}$for $C_{q}^{+}, C_{q}^{-}, D_{q}^{+}$, and $D_{\bar{q}}^{-}$whenever it is clear to which arc we are referring.

PROOF OF THEOREM.

Definition. $\bar{C}$ belongs to Class II if and only if an infinite $W$-characteristic or a closed $W$-characteristic belongs to $\bar{C}$. Otherwise $\bar{C}$ belongs to Class I.

LEMmA I. Let $A B$ be an arc on $M$ which is part of a $V$-characteristic at a finite distance from the singular points of $V$ and which does not form a closed curve. Let $A B$ carry a strictly increasing continuous parameter $\tau, 0 \leqq \tau \leqq \sigma$. Let the orthogonal trajectory through $A$ carry a strictly increasing continuous parameter $\rho$ in some neighborhood of $A$ such that

Presented to the Society, December 29, 1952 under the title $A$ theorem about differential equations on two-dimensional manifolds; received by the editors January 12 , 1953.

1 The author wishes to express his appreciation to Professors Witold Hurewicz and Norman Levinson of the Massachusetts Institute of Technology who have given valuable advice, and to the Office of Naval Research for financial support.

A point $p$ is an $\omega$-limit point of $C^{+}$on $M$ if for every $\epsilon>0$ and every $q$ belonging to $C^{+}$there exists a point $r$ following $q$ on $C^{+}$which is within $\epsilon$ of $p$. 
$\rho(a)=0$. Then there exists $\epsilon>0$ and $a$ map $\phi$ of the rectangle $\{(\rho, \tau)|| \rho \mid$ $<\epsilon, 0 \leqq \tau \leqq \sigma\}$ into $M$ such that (1) $\phi$ is a homeomorphism, (2) $\phi(0, \tau)$ is the point on arc $A B$ with parametric value $\tau,(3) \phi(\rho, 0)$ is the point on $D_{A^{+}}$or $D_{-}^{-}$, depending on whether $\rho \geqq 0$ or $\rho \leqq 0$, with parametric value $\rho,(4) \phi(\rho, \tau) \in D_{\phi(0, \tau)}^{+} \cap C_{\phi(\rho, 0)}^{+}$or $D_{\phi(0, \tau)}^{-} \cap C_{\phi(0,0)}^{+}$depending on whether $\rho \geqq 0$ or $\rho \leqq 0$.

Lemma I follows from the fundamental existence theorem and some elementary trigonometric considerations.

Definition. A map $\phi$ of $\{(\rho, \tau) \mid \epsilon>\rho>-\epsilon, 0 \leqq \tau \leqq \sigma\}$ of the type described above which sends $\{(\rho, \tau) \mid \rho=0,0 \leqq \tau \leqq \sigma\}$ into an arc $A B$ will be called a "net map" with $A B$ the "core" of the net map.

We first prove that if $\bar{C}$ belongs to Class $I$ then $\bar{C}$ is nowhere dense. This proof will proceed by contradiction. Let $p$ be an interior point of $G$, the set in which $\bar{C}$ is dense. Then $p$ is an interior point of $\bar{C}$ since all of $G$ belongs to $\bar{C}$ which is a closed point set. Let $D$ be the orthogonal characteristic through $p, E$ the intersection of $\bar{C}$ and $D$, and $K$ the component of $E$ in the one-dimensional topology of $D$ to which $p$ belongs. $K$ is an interval, since $\bar{C}$ belongs to Class $I$, and is closed by the properties of $\bar{C}$. In what follows we may assume that $p$ belongs to $C^{+}$since the interior of $K$ must contain points of $C^{+} . C_{p}^{+}$ then must return to the interior of $K$ since $K \subset \bar{C}$.

Lemma II. Assuming that $\bar{C}$ belongs to Class I and that $p$ and $K$ have the same meaning as above we let $p^{*}$ be the first intersection of $C_{p}^{+}$with $K$. $\rho$ and $\tau$ are continuous strictly increasing parameters on $K$ and on the arc $p p^{*}$ respectively with $\rho(p)=\rho_{0}, \tau(p)=0, \tau\left(p^{*}\right)=1, \rho$ (the end point of $K$ on $\left.D_{p}^{+}\right)=1$, and $\rho$ (the end point of $K$ on $\left.D_{p}^{-}\right)=0$. Then there exists a topological space $X$ and a map $\phi$ of $X$ into $M$ such that (1) $X$ is obtained from the unit square by identifying the edge $\tau=1$ with a closed subinterval of $\tau=0$ in a sense-preserving manner, (2) $\phi$ is a homeomorphism, (3) $\phi(\rho, 0)$ is the point on $K$ with parametric value $\rho$, (4) $\phi\left(\rho_{0}, \tau\right)$ is the point on the arc $p p^{*}$ with parametric value $\tau,(5) \phi(\rho, \tau)$ belongs to $C_{\rho}^{+} \cap D_{\tau}^{+}$or $C_{\rho}^{+} \cap D_{\tau}^{-}$depending on whether $\rho \geqq 0$ or $\rho \leqq 0$.

Proof of Lemma II. Let $J$ be the maximal interval around $\rho_{0}$ in $(0 \leqq \rho \leqq 1)$ such that the set $\{(\rho, \tau) \mid \rho \in J, 0 \leqq \tau \leqq 1\}$ is a subset of a space $X$ described in the statement of Lemma II and such that there exists a map $\phi$ of this set into $M$ which satisfies conditions 1-5 and such that $\phi(\rho, \tau)$ belongs to $K$ if and only if $\tau=0$ or $\tau=1$. If we can show that $J$ is the whole unit interval we have proved the lemma.

First $J$ is open by Lemma $\mathrm{I}$.

Next we show that $J$ is also closed. Let $J_{\tau_{0}}=\phi\left\{\left(\rho, \tau_{0}\right) \mid \rho \in J\right.$ and $\tau_{0}$ is fixed $\}$. Since we assumed that $\bar{C}$ belongs to Class $I, J_{\tau_{0}}$ is a finite 
interval on an orthogonal trajectory. If $J$ is not closed let $\bar{\rho}$ be an end point of $J$ which does not belong to $J$. Let $\Gamma\left(\bar{\rho}, \tau_{0}\right)$ be the corresponding end point of $J_{\tau_{0}}$. We assume that $\bar{\rho}>\rho_{0}$ and that hence $\Gamma\left(\bar{\rho}, \tau_{0}\right) \in D_{\tau_{0}}^{+}$; the other case can be handled identically.

We note that in Lemma I we could have let $A B$ be an arc on a $W$-characteristic instead of on a $V$-characteristic. Hence, according to Lemma I there exists an $\epsilon\left(\tau_{0}\right)$ and a net map $\psi_{\tau_{0}}$ which has the arc $\phi\left(\rho_{0}, \tau_{0}\right) \Gamma\left(\bar{\rho}, \tau_{0}\right)$ as a core such that $\psi_{\tau_{0}}\left(\rho, \tau_{0}\right)=\phi\left(\rho, \tau_{0}\right)$ for $\rho_{0} \leqq \rho<\bar{\rho}$ and such that $\psi_{\tau_{0}}\left(\rho_{0}, \tau\right)=\phi\left(\rho_{0}, \tau\right)$ for $\left|\tau-\tau_{0}\right|<\epsilon\left(\tau_{0}\right)$. We claim that $\psi_{\tau_{0}}=\phi$ for the intersection of domains. This is true for $\left\{\left(\rho, \tau_{0}\right) \mid \rho_{0}\right.$ $\leqq \rho<\bar{\rho}\}$ and $\left\{\left(\rho_{0}, \tau\right)|| \tau-\tau_{0} \mid<\epsilon\right\}$ by the construction of the net map. If $\tau>\tau_{0}, \psi_{\tau_{0}}(\rho, \tau)$ is just the first intersection of $C_{\phi_{\tau_{0}}\left(\rho, \tau_{0}\right)}^{+}$(first on $\left.C_{\phi_{\tau} 0\left(\rho, \tau_{0}\right)}^{+}\right)$with $D_{\phi_{\tau_{0}}\left(\rho_{0}, \tau\right)}^{+}$before $D_{\phi_{0}\left(\varphi_{0}, \tau\right)}^{+}$returns to $\psi_{\tau_{0}}\left\{\left(\rho_{0}, \tau\right)|| \tau-\tau_{0} \mid\right.$ $<\epsilon\}$; on the other hand the same statement holds for $\phi(\rho, \tau)$ with $C_{\phi\left(\rho, \tau_{0}\right)}^{+}$taking the place of $C_{\phi_{\tau_{0}}\left(\rho, \tau_{0}\right)}^{+}$and $D_{\phi\left(\rho_{0}, \tau\right)}^{+}$taking the place of $D_{\phi_{\tau_{0}}\left(\rho_{0}, \tau\right)}^{+}$. Since it has been previously remarked that $\phi\left(\rho, \tau_{0}\right)$ $=\pi_{\tau_{0}}\left(\rho, \tau_{0}\right)$ and $\phi\left(\rho_{0}, \tau\right)=\psi_{\tau_{0}}\left(\rho_{0}, \tau\right)$ we have that $\phi=\psi_{\tau_{0}}$ for the intersection of domains and $\tau \geqq \tau_{0}$. It is shown in the same way that $\phi=\psi_{\tau_{0}}$ for the intersection of domains and $\tau<\tau_{0}$. A finite number of the $\epsilon\left(\tau_{0}\right)$ cover the range $0 \leqq \tau \leqq 1$ by compactness. One thus finds that $\Gamma(\bar{\rho}, \tau)$ belongs to $C_{\bar{\rho}}^{+} \cap D_{\tau}^{+}$for all $\tau$. We define $\phi(\bar{\rho}, \tau)$ to equal $\Gamma(\bar{\rho}, \tau)$.

The proof that the thus extended map is a homeomorphism and satisfies all the properties required of $\phi$ follows:

(1) The extended map is continuous in view of the continuity of the local maps.

(2) The extended map is one-to-one and $\phi(\rho, \tau)$ belongs to $K$ if and only if $\tau=0$ or 1 .

(a) $\phi\left(\bar{\rho}, \tau_{1}\right) \neq \phi\left(\bar{\rho}, \tau_{2}\right)$ if $\left(\tau_{1}, \tau_{2}\right) \neq(0,1)$.

This follows from the fact that $\phi\left(\bar{\rho}, \tau_{1}\right)$ is the upper end point of $J_{\tau_{1}}$ (upper in the sense of the orthogonal trajectory) and that $\phi\left(\bar{\rho}, \tau_{2}\right)$ is the upper end point of $J_{\tau_{2}}$ which two sets do not intersect by hypothesis on $J$.

(b) If $\phi(\bar{\rho}, 0)=\phi(\bar{\rho}, 1)$ the extended map can still be made one-toone.

For in this case, for some $\eta$ and $\mu, \phi\{(\rho, 0) \mid \bar{\rho}-\eta \leqq \rho<\bar{\rho}\}=\phi\{(\rho, 1) \mid \bar{\rho}$ $-\mu \leqq \rho<\bar{\rho}\}$ on $M$. This in turn implies that $\{(\rho, 0) \mid \bar{\rho}-\eta \leqq \rho<\bar{\rho}\}$ $=\{(\rho, 1) \mid \bar{\rho}-\mu \leqq \rho<\bar{\rho}\}$ in $X$. We can thus keep the map one-to-one by identifying $(\bar{\rho}, 0)$ with $(\bar{\rho}, 1)$ in the extended $X$.

(c) $\phi / \bar{\rho}$ is a homeomorphism in the one-dimensional topology since it is a one-to-one continuous map of a compact Hausdorff space into a Hausdorff space.

(d) $\phi(\bar{\rho}, \tau)$ belongs to $K$ if and only if $\tau=0$ or 1 . 
Suppose $\phi\left(\bar{\rho}, \tau_{0}\right) \in K$ for $\tau_{0} \neq 0$ or 1. Then $J_{\tau_{0}}$ belongs to $K$ also if it only belongs to $\bar{C}$ since $K$ is a component of $\bar{C}$ on a certain orthogonal characteristic. But all points of $J_{\tau_{0}}$ are on $V$-characteristics starting at points of $K$ which automatically belong to $\bar{C}$, and hence $J_{\tau_{0}}$ belongs to $\bar{C}$. On the other hand, $J_{\tau_{0}}$ does not belong to $K$ if $\tau_{0} \neq 0$ or 1 . Hence, $\phi\left(\bar{\rho}, \tau_{0}\right)$ cannot belong to $K$ if $\tau_{0}$ is different from 0 and 1 . $\phi(\bar{\rho}, 0)$ and $\phi(\bar{\rho}, 1)$ belong to $K$ since they belong to $\bar{C}$, and $J_{0}$ and $J_{1}$ belong to $K$.

(e) $\phi\left(\bar{\rho}, \tau_{1}\right) \neq \phi\left(\rho, \tau_{2}\right)$ for $\rho \in J$ and $\tau_{1} \neq \tau_{2}$.

If this statement were false we would have either of two possibilities: $\phi(\bar{\rho}, 0)$ would be an interior point of the $\operatorname{arc} \phi(\rho, 0) \phi\left(\rho, \tau_{2}\right)$ or $\phi(\rho, 0)$ would be an interior point of the $\operatorname{arc} \phi(\bar{\rho}, 0) \phi\left(\bar{\rho}, \tau_{1}\right)$. But neither of these arcs can contain any interior points belonging to $K$. This is true for the first arc since $\rho \in J$ and for the second arc by part (d).

(f) $\phi\left(\bar{\rho}, \tau_{0}\right) \neq \phi\left(\rho, \tau_{0}\right)$ for $\rho \in J$.

If this statement were false $\bar{C}$ would contain a closed orthogonal characteristic which is contrary to hypothesis.

(3) The extended map is a homeomorphism.

We have shown that $\phi$ extended is one-to-one and continuous. If $J$ had been open at both end points we could have extended the map $\phi$ similarly in the other direction; if $J$ had been open only at one end $(\bar{\rho})$ this would not have been necessary. In either case we would obtain a one-to-one continuous map of a compact Hausdorff space into a Hausdorff space. The inverse continuity then follows automatically.

Thus $\phi$ can be extended. $J$ is not maximal, which is a contradiction. Hence $J$ is closed. This concludes the proof of Lemma II.

\section{Lemma III. If $\bar{C}$ is dense in some set $G$ of $M$, then $\bar{C}$ cannot belong to Class I.}

Proof of Lemma III. Let $p, p^{*}, D$, and $K$ have the same meaning as before. By Lemma II all the $V$-characteristics starting at $K$ return to $K$ and the map *, which is defined for all points of $K$ by $q^{*}$ is the first intersection of $C_{q}^{+}$with $K$, is a sense-preserving homeomorphism. ( $q$ is an arbitrary point of $K$.) It has previously been pointed out that we may assume that $p$ belongs to $C^{+}$. Hence $p^{*}$ cannot equal $p$; for if it $\operatorname{did} C^{+}$would be a closed curve and all of $K$ would not belong to $\bar{C}$. On the other hand, if $p^{*} \neq p$, in view of the sense-preserving nature of the transformation ${ }^{*}$, successive intersections of $C_{p}^{+}$would all intersect $K$ on the same side of $p$. This contradicts the fact that there are $\omega$-limit points of $C^{+}$on the other side of $p$.

Lemma IV. If $\bar{C}$ belongs to Class II, then there exists a closed curve $S$ 
with continuous curvature such that the solution curves of $V$ are never tangent to $S$ and always cross $S$ in the same direction and such that $S$ belongs to $\bar{C}$.

Proof of Lemma IV. This is proved very much like a similar lemma of Siegel's [3]. It is obviously true if a closed curve characteristic of the orthogonal vector field belongs to $\bar{C}$. Otherwise let $\bar{b}$ be an $\omega$-limit point of the infinite orthogonal characteristic $D$ belonging to $\bar{C}$ on the compact set $M$. Let $O$ be a neighborhood of $b$ in which $V$ varies by less than $\pi / 16$ radians; let $P$ and $Q$ be the neighborhoods of $b$ with diameter one-half the diameter of $O$ and one-quarter the diameter of $O$ respectively. Since $\bar{C}$ is a closed point set, $b \in \bar{C}$ and thus is at a finite distance from the singular points of $V$; hence a set $O$ of the type described can always be constructed.

Let $L_{1}$ and $L_{2}$ be line segments through $\delta$ in $Q$ which make angles of $45^{\circ}$ and $135^{\circ}$ respectively with the direction of $V$ at $b . D$ will intersect these line segments again and again. We shall say that $c$ is an intersection of $D$ with $L_{2}$ which corresponds to an intersection $b$ of $D$ with $L_{1}$ if the $\operatorname{arc} b c$ on $D$ is entirely contained in $P$. The limited variation of $V$ in this neighborhood assures us that for a given $b$ there is exactly one $c$ and conversely. Let $b_{1}$ and $b_{2}$ be successive intersections of $D$ with $L_{1}$, and $c_{1}$ and $c_{2}$ the corresponding intersections with $L_{2}$. The choice of $O, P$, and $Q$ implies that the arc $b_{1} b_{2}$ and $c_{1} c_{2}$ on $D$ contain points outside $O$. The limited variation of $V$ in $O$ also implies that $V$-characteristics starting on the line segments $b_{1} b_{2}$ and $c_{1} c_{2}$ on $L_{1}$ and $L_{2}$ respectively intersect $D$ before leaving $O$. Thus, these two line segments belong to $\bar{C}$. Let $T$ be the curve formed by the $\operatorname{arc} b_{1} b_{2}$ on $D$ and the line segment $b_{1} b_{2}$ on $L_{1}$. Let $T^{\prime}$ be the curve formed by the arc $c_{1} c_{2}$ on $D$ and the line segment $c_{1} c_{2}$ on $L_{2}$. Then except for the condition of continuous curvature either $T$ or $T^{\prime}$ satisfies the lemma. It has been shown that both $T$ and $T^{\prime}$ belong to $\bar{C}$ and the $V$-characteristics either cross the line segment $b_{1} b_{2}$ or the line segment $c_{1} c_{2}$ in the same direction in which they cross the part of $D$ which runs through $b_{1} c_{1} b_{2} c_{2}$. Finally, the chosen curve, whether $T$ or $T^{\prime}$, can always be given continuous curvature, as Siegel remarks in the abovementioned paper. In what follows we shall assume that $T$ is the chosen curve and $b_{1}$ and $b_{2}$ are the points which have been connected by a straight line.

Lemma V. If $\bar{C}$ is dense in some set of $M$ then $\bar{C}$ cannot belong to Class II unless $M$ is a torus and $V$ is free of singular points.

Proof of Lemma V. In the proof of this lemma we make use of the closed curve constructed in Lemma IV. We first define parallels 
to this closed curve. These parallels are generally just orthogonal trajectories, but they reduce to straight lines in the neighborhood of the straight line part of $S$.

Definition. Let $b_{1}, b_{2}, O, P$, and $Q$ have the same meaning as in Lemma IV. Let $d_{2} \in C_{b_{2}}^{+}$and $e_{1} \in C_{b_{1}}^{-}$be arbitrary points such that the $\operatorname{arcs} b_{2} d_{2}$ on $C_{b_{2}}^{+}$and $b_{1} e_{1}$ on $C_{b_{1}}^{-}$are contained in $P$. Then in view of the limited variation of $V$ and hence $W$ in $O$ there exist points $d_{1} \in C_{b_{1}}^{+} \cap D_{d_{2}}^{+}$and $e_{2}$ on $C_{b_{2}}^{-} \cap D_{e_{1}}^{-}$such that the arcs $b_{1} d_{1}$ on $C_{b_{1}}^{+}, d_{2} d_{1}$ on $D_{d_{2}}^{+}, b_{2} e_{2}$ on $C_{b_{2}}^{-}$, and $e_{1} e_{2}$ on $D_{e_{1}}^{-}$are all contained in $O$. If $x$ is outside the quadrilateral $d_{1} d_{2} e_{1} e_{2}$, then the parallel to $S$ through $x$ is just the orthogonal trajectory. To define the parallels inside $d_{1} d_{2} e_{1} e_{2}$ we consider an arbitrary point $r$ on the line segment $b_{1} b_{2}$ on $L_{1}$. $C_{r}^{+}$intersects $d_{1} d_{2}$ at a point $s$ and $C_{r}^{-}$intersects $e_{1} e_{2}$ at a point $t$. We parametrize the arc st on $C_{t}^{+}$by a parameter proportional to the arc length such that the parametric value of $t$ is zero, the parametric value of $r$ is one, and the parametric value of $s$ is two. Inside the quadrilateral $d_{1} d_{2} e_{1} e_{2}$ the parallels will just be the lines of equal parameter.

We next prove that all $V$-characteristics starting on $S$ return to $S$, and that, in fact, a torus obtained from the unit square by double identification can be mapped homeomorphically onto the point set generated by these characteristics.

The proof of this statement follows very closely the proof of Lemma II. We again pick a point $p$ on $S$ which belongs to $C^{+}$. We again define parameters $\rho$ and $\tau$ such that $\rho(p)=\rho_{0}$, but now the points with parametric values 0 and 1 coincide on $S$. The parallels defined above take the place of orthogonal trajectories. There are two differences in the proof. First of all we must now show that after the map $\phi$ has been extended to $\rho=0$ and $\rho=1$, the set $\phi\{(0, \tau) \mid 0 \leqq \tau \leqq 1\}$ equals the set $\phi\{(1, \tau) \mid 0 \leqq \tau \leqq 1\}$. The second and major difference, however, is due to the fact that in the proof of Lemma II, in the part where $J$ was proved to be closed, we made use of the fact that $\bar{C}$ belongs to Class I and that hence $J_{\tau_{0}}$ is a finite interval. We must now show independently that if $J$ is not the whole unit interval, $J_{\tau_{0}}$ cannot be of infinite arc length.

The first difference is disposed of easily. First, $\phi(0,0)$ equals $\phi(1,0)$ since the points with parametric values 0 and 1 on $S$ coincide. $\phi(0,1)$ is the first intersection of $C_{\phi(0,0)}^{+}$with $S$ after $\phi(0,0) ; \phi(1,1)$ is the first intersection of $C_{\phi(1,0)}^{+}$with $S$ after $\phi(1,0)$. Hence, $\phi(0,1)=\phi(1,1)$, and the $\operatorname{arcs} \phi(0,0) \phi(0,1)$, and $\phi(1,0) \phi(1,1)$ coincide.

We resolve the second difficulty by a proof using a contradiction. Suppose that $J_{\tau_{0}}$ is of infinite arc length and that $J$ is not the whole unit interval. This means, in particular, that the image under $\phi$ of 
the set $\{(\rho, 0) \mid \rho \in J\}$ is not all of $S$. We can construct a closed curve $T$ from $J_{r_{0}}$ and an arc of arbitrarily short length just as in Lemma IV. Let $c_{1}$ and $c_{2}$ be the points on $J_{\tau_{0}}$ which are connected by a line segment. (We may assume that the line segment chosen makes an angle of between $40^{\circ}$ and $50^{\circ}$ with the vectors of $V$ and of $W$ at $c_{1}$ and $c_{2}$.) Then $C_{c_{1}}^{+}$or $C_{c_{1}}^{-}$will intersect the $W$-characteristic through $c_{2}$ at a point $c_{3}$, and the arc length of the $\operatorname{arc} c_{1} c_{3}$ on the $V$-characteristic will be less than four times the length of the segment $c_{1} c_{2}$. If we use $\phi$ for the map from the torus or its subset determined by $J$ into $M$, then $c_{1}=\phi\left(\rho_{1}, \tau_{0}\right)$ and $c_{3}=\phi\left(\rho_{2}, \tau_{0}\right)$ for some $\rho_{1}$ and $\rho_{2}$. The nature of $\phi$ is such that if $c_{3}$ belongs to $C_{c_{1}}^{+}$then $\phi\left(\rho_{2}, 0\right)$ lies between $\phi\left(\rho_{1}, \tau_{0}\right)$ and $\phi\left(\rho_{2}, \tau_{0}\right)$, and if $c_{3}$ belongs to $C_{c_{1}}^{-}$then $\phi\left(\rho_{1}, 0\right)$ lies between $\phi\left(\rho_{2}, \tau_{0}\right)$ and $\phi\left(\rho_{1}, \tau_{0}\right)$. In either case we can show that for every $\epsilon>0$ there exists $\rho(\epsilon)$ belonging to $J$ such that the $\operatorname{arc} \phi(\rho(\epsilon), 0) \phi\left(\rho(\epsilon), \tau_{0}\right)$ is shorter than $\epsilon$. On the other hand, if we use the fact that there can be no infinite parallel to $S$ near $S$ we can show that there exists $\epsilon_{0}>0$ such that the $\operatorname{arcs} \phi(\rho, 0) \phi\left(\rho, \tau_{0}\right)$ are longer than $\epsilon_{0}$ for all $\rho$ belonging to $J$. Hence, $J_{\tau_{0}}$ cannot be of infinite arc length.

Once we have proved that a torus can be mapped into $M$ in the indicated manner Lemma $\mathrm{V}$ is proved.

Lemmas III and V prove the theorem.

\section{REFERENCES}

1. F. Haas, Proc. Nat. Acad. Sci. U.S.A. vol. 38 (1952) pp. 1044-1047.

2. A. Denjoy, Sur les courbes définies par les équations différentielles a la surface du tore, J. Math. Pures appl. (9) vol. 11 (1932) pp. 333-375.

3. C. L. Siegel, Note on differential equations on the torus, Ann. of Math. (3) vol. 46 (1945) pp. 423-427.

LEHIGH UNIVERSITY 